\title{
Comprehensive circular RNA expression profiling with associated ceRNA network reveals their therapeutic potential in cholesteatoma
}

\author{
JUANJUAN GAO ${ }^{1-3}$, QI TANG ${ }^{2,3}$, RUOYAN XUE ${ }^{2,3}$, XIAOHUI ZHU ${ }^{2,3}$, SHIHUA WANG $^{4}$, \\ YONGLI ZHANG ${ }^{2,3}$, WENBIN LIU ${ }^{2,3}$, ZHIQIANG GAO ${ }^{2,3}$ and HUA YANG ${ }^{2,3}$ \\ ${ }^{1}$ Department of Otolaryngology, Head and Neck Surgery, Beijing Tsinghua Changgung Hospital,
} School of Clinical Medicine, Tsinghua University, Beijing 102218; ${ }^{2}$ Department of Otolaryngology; ${ }^{3}$ Translational Medicine Center, Peking Union Medical College Hospital, Chinese Academy of Medical Sciences and Peking Union Medical College; ${ }^{4}$ Center of Excellence in Tissue Engineering, Key Laboratory of Beijing, Institute of Basic Medical Sciences and School of Basic Medicine, Chinese Academy of Medical Sciences and Peking Union Medical College, Beijing 100730, P.R. China

Received August 7, 2019; Accepted January 14, 2020

DOI: $10.3892 /$ or.2020.7501

\begin{abstract}
Cholesteatoma is a chronic disease that pathologically displays a benign tumor with excessive squamous epithelial cell proliferation in the middle ear. Clinically, however, it can manifest malignant behavior by destroying adjacent tissues and organs. Although previous studies have demonstrated that the pathogenesis of cholesteatoma is correlated with epigenetic dysregulation, the exact mechanism remains unclear. Circular RNAs (circRNAs) have been revealed as being abundantly expressed in various organisms and have been found to contribute to the regulation of many diseases. To date, no reports have elucidated their expression profiles and functions in cholesteatoma. In the present study, the circRNA expression profile in cholesteatoma was explored for the first time by using microarray analysis. We obtained a total of 355 significantly differentially expressed circRNAs in cholesteatoma, among which 101 were identified to be upregulated and 254 downregulated. By constructing circRNA-lncRNA-miRNA-mRNA competing endogenous RNA (ceRNA) network, it was discovered that circRNAs may function as ceRNAs and contribute to the formation of cholesteatoma. These results provide novel insight into the pathogenesis of cholesteatoma and suggest circRNAs as potential promising therapeutic targets for cholesteatoma.
\end{abstract}

Correspondence to: Dr Hua Yang, Department of Otolaryngology, Peking Union Medical College Hospital, Chinese Academy of Medical Sciences and Peking Union Medical College, Beijing 100730, P.R. China

E-mail: yanghua702@163.com

Key words: cholesteatoma, circular RNA, circRNA, microarray analysis, competing endogenous RNA, network

\section{Introduction}

Cholesteatoma is a chronic middle ear disease, which is pathologically displayed as a benign tumor with excessive squamous epithelial cell proliferation. Nevertheless, it can clinically manifest a malignant nature by destroying adjacent bony structures and nerves, resulting in hearing loss, tinnitus, dizziness, facial paralysis, brain abscess, meningitis, and hydrocephalus (1). To date, the only treatment for cholesteatoma is surgical resection as no drug treatments are currently available, although recurrence with complications after surgery is quite high (2).

Previous reports have indicated that the underlying molecular mechanisms of cholesteatoma are regulated by growth factors and inflammatory mediators (3-6). In recent years, epigenetic regulation, such as dysregulation of microRNAs (miRNAs), has been demonstrated to play a crucial role in cholesteatoma formation (7-11). Most experiments have focused on discovering the messenger RNA (mRNA) targets of miRNAs and elucidating their regulatory mechanisms on the assumption that following buffering of the expression of an miRNA, the expression of targeted mRNAs is correspondingly disturbed $(7,8)$. However, a single miRNA can regulate the expression of hundreds of mRNAs and each mRNA can be regulated by several miRNAs (12), all of which complicate the functional research of the molecular mechanism of cholesteatoma.

Recent years, in the research of therapeutic targets of diseases, endogenous miRNA 'sponges', also termed competing endogenous RNAs (ceRNAs), which contain tandem repeats of miRNA recognition elements (MREs), have shed new light on the investigation of the function of miRNAs $(13,14)$. ceRNAs can act as natural miRNA sponges and thus influence the expression of miRNAs. All RNA transcripts that share common MREs can function as ceRNAs, such as protein coding genes, pseudogenes, long non-coding RNAs (lncRNAs) $(13,15)$, and recently discovered circular RNAs (circRNAs) (16).

In our previous study, we reported that IncRNAs had ceRNA potential in cholesteatoma formation (15). Unlike 
lncRNAs, circRNAs are a novel class of noncoding RNAs characterized by the unique structure wherein the $3^{\prime}$ and 5 ends are covalently joined in a closed loop structure without polarity or poly (A) tail $(17,18)$. The special structure ensures the much higher stability of circRNAs than linear transcripts, protecting circRNAs from exonucleolytic decay $(16,19,20)$. Studies have shown that circRNAs can function as endogenous miRNA sponges, which can efficiently soak up miRNAs and buffer their activities, resulting in the upregulated expression of miRNA-targeted genes (21). The circRNA-mediated ceRNA network has been verified to play crucial roles in many disease processes, such as bladder cancer (22), cardiovascular diseases (23), and Alzheimer's disease (24). Despite the marked regulatory potential in disease, however, no reports have elucidated whether circRNAs can play a role in cholesteatoma.

In our present study, we explored the differentially expressed profile of circRNAs between cholesteatoma and matched normal skin tissues by using microarray analysis for the first time. The reliability of microarray expression data was confirmed by quantitative RT-PCR. By constructing the circRNA-lncRNA-miRNA-mRNA ceRNA network with bioinformatics approaches, we explored the ceRNA potential of circRNAs in the pathogenesis of cholesteatoma.

\section{Materials and methods}

Patients and specimens. All specimens were obtained from 3 female and 4 male patients aged 18 to 32-years-old (mean age 26.3 years, 2 female and 2 male patients for microarray analysis and real-time qPCR validation, 1 female and 2 male patients for real-time qPCR validation), who underwent surgical procedures for unilateral middle ear cholesteatoma between June 2016 and November 2016 at the Department of Otorhinolaryngology, Peking Union Medical College Hospital, Beijing, China. All patients in this study met the following inclusion criteria: Patients presented with acquired cholesteatoma, the resected mass was identified as cholesteatoma by pathological examination, and no antitumor treatments were given before surgery. Patients that had previous middle ear surgeries or combined with other middle ear tumors were excluded. All specimens were stored at $-80^{\circ} \mathrm{C}$ after collection for subsequent RNA extraction.

RNA extraction and quality control. Total RNAs were extracted from cholesteatoma and post-auricular skin tissues using TRIzol reagent (Invitrogen; Thermo Fisher Scientific, Inc.) according to the manufacturer's instructions. The concentrations of the RNA samples were determined by OD260 using a NanoDrop ND-1000 instrument (Thermo Fisher Scientific, Inc.). The integrity of RNAs was assessed by electrophoresis on a denaturing agarose gel and with an Agilent 2100 Bioanalyzer (Agilent Technologies, Inc.).

Microarray assay. Microarray analysis was used to detect differentially expressed circRNAs between cholesteatoma and post-auricular skin tissues. Sample labelling and array hybridization were performed according to the manufacturer's protocol (Arraystar Inc.). The sample preparation and microarray hybridization were performed based on the Arraystar's standard protocols including RNA purification, amplification, and transcription into fluorescent cRNA. The labelled cRNAs were then hybridized onto an assembled RNA expression microarray slide (Arraystar). After washing, the arrays were scanned using the Agilent G2505C Scanner. Agilent Feature Extraction software (version 11.0.1.1) was used to analyze acquired array images. Quantile normalization and subsequent data processing were performed using the R software limma package (version 3.22.7) (25) and GeneSpring GX v12.1 (Agilent Technologies). Differentially expressed circRNAs between two samples were identified through fold change filtering. Hierarchical clustering was performed to show the distinguishable RNA expression patterns among the samples.

Real-time $q P C R$ validation. Extracted RNA was reverse transcribed to synthesize cDNA for RT-qPCR analysis. RNA (3 $\mu \mathrm{g})$ was mixed with $1 \mu \mathrm{l}$ Random N9 primers $(0.5 \mu \mathrm{g} / \mu \mathrm{l})$ (Invitrogen; Thermo Fisher Scientific, Inc.) and $1.6 \mu \mathrm{ldNTP}$ Mix (HyTest Ltd.), and then the mixture was put on ice for $2 \mathrm{~min}$ followed by incubation at $65^{\circ} \mathrm{C}$ for $5 \mathrm{~min}$. The reverse transcription system was subsequently prepared, comprising the above mixture, $0.2 \mu \mathrm{l}$ SuperScript III RT (Invitrogen; Thermo Fisher Scientific, Inc.), $4 \mu 1$ XX First-Strand Buffer (Invitrogen; Thermo Fisher Scientific, Inc.), $1 \mu 10.1 \mathrm{M}$ DTT (Promega) and $0.3 \mu \mathrm{l}$ RNase inhibitor (Epicentre, Inc.). This reaction system underwent successive incubation in water at a temperature of $37^{\circ} \mathrm{C}$ for $1 \mathrm{~min}, 50^{\circ} \mathrm{C}$ for $60 \mathrm{~min}$ and then $70^{\circ} \mathrm{C}$ for $15 \mathrm{~min}$ until reverse transcription was completed. The selected circRNAs and primers for RT-qPCR were designed using Primer 5.0 software (Primer-E Ltd., UK) (Table I) and synthesized by Generay Biotech. For all samples, $\beta$-actin was used as an internal control. RT-qPCR was performed using the ViiA 7 Real-time PCR System (Applied Biosystems; Thermo Fisher Scientific, Inc.) with a SYBR expression assay system (Takara). The PCR reaction conditions were as follows: An initial denaturation at $95^{\circ} \mathrm{C}$ for $10 \mathrm{~min}$, followed by 40x PCR cycles at $95^{\circ} \mathrm{C}$ for $10 \mathrm{sec}$ and $60^{\circ} \mathrm{C}$ for $60 \mathrm{sec}$, then annealing and extension at $95^{\circ} \mathrm{C}$ for $10 \mathrm{sec}, 60^{\circ} \mathrm{C}$ for $60 \mathrm{sec}$, and finally $95^{\circ} \mathrm{C}$ for $15 \mathrm{sec}$. Each sample was assayed in triplicate. The $2^{-\Delta \Delta \mathrm{Cq}}$ method was used to determine the relative expression level of each circRNA (26). We used an unpaired t-test to compare the expression of circRNAs between cholesteatoma and normal skin samples. A P-value $<0.05$ was considered to be statistically significant.

$G O$ and KEGG pathway analyses. Gene ontology (GO) provides a 'framework for the model of biology' (http://www. geneontology.org). The ontology describes the genes, gene product functions, and their inter-relationships. It classifies functions into three aspects, biological process (BP), cellular component (CC) and molecular function (MF). Fisher's exact test was used to elucidate the overlap between the gene list and the GO annotation list. The - $\log 10$ (P-value) was applied to denote the significance of the GO term enrichment in the analyzed genes. A lower P-value indicated a more significant GO term (recommended P-value <0.05). Pathway analysis was performed to predict molecular interactions and reaction networks by mapping genes to Kyoto Encyclopedia of Genes and Genomes (KEGG) (http://www.genome.jp/kegg/). The $-\log 10$ (P-value) was used to denote the significance of 
Table I. Primers for RT-qPCR analysis.

circRNAs

Primer sequences

hsa_circRNA_006562

hsa_circRNA_084725

hsa_circRNA_101458

hsa_circRNA_101965

hsa_circRNA_102747

hsa_circRNA_103276

$\beta$-actin
F: 5'-ACGAGAAGACCCGCAAGATTAC-3

R: 5'-GCGTTCAGACCTAAGGCTCATC-3'

F: 5'-GTAACACTCAGGTCCGTAGAAGA-3'

R: 5'-CAGACTGGCTCATACTCGTGT-3'

F: 5'-TTTAGACCGTCTGGCTACACC-3'

R: 5'-CGTTCTGGGTTGATTCTGTTC-3'

F: 5'-CATCCGATCCAGGTGTTTTAC-3'

R: 5'-TCAGAAACTTGATCCTGGTGTCT-3'

F: 5'-TGTGCTTTCTGGAGGGTCTACT-3'

R: 5'-TGCCTCATCACCAACCATAAG-3'

F: 5'-TGTTTTCACCAGTCACATCTCTT-3'

R: 5'-CCCAGCCCTCAGTTGTATTC-3'

F: 5'-GTGGCCGAGGACTTTGATTG-3'

R: 5'-CCTGTAACAACGCATCTCATATT-3'

circRNAs, circulating RNAs; F, forward; R, reverse.

the pathway correlations, wherein a lower P-value indicated a more significant correlation (recommended P-value $<0.05$ ).

ceRNA network analysis. Significantly differentially expressed circRNAs were subjected to ceRNA network analysis. The potential miRNA recognition elements (MREs) were predicted based on the sequences of circRNAs and mRNAs. miRNA binding seed sequence sites were predicted using miRanda (27) (http://www.microrna.org/microrna/) and TargetScan (28) (http://www.targetscan.org/). Then miRNAs were optimized and selected by parameter settings of Context $\leq-0.10$ and Context ${ }^{+} \leq-0.10$. ceRNAs were filtered based on matching abilities.

Statistical analysis. In the microarray data analysis, when comparing two groups of profile differences, the statistical significance of the difference was estimated using an unpaired t-test. SPSS 20.0 software (IBM, Inc.) was used for statistical analysis. In the RT-qPCR validation, the data are expressed as the means \pm SE using GraphPad Prism 6.05 software (GraphPad Software, Inc.). Unpaired t-tests were used to compare the expression of circRNAs between two groups. $\mathrm{P}<0.05$ was considered to indicare a statistically significant difference.

\section{Results}

Significant differential expression profiles of circRNAs are found in cholesteatoma compared to matched normal skin tissues. In the present study, a total of 13,247 circRNAs were detected by microarray analysis. By comparing cholesteatoma and normal skin groups, circRNA expression patterns between the groups were elucidated as being quite different. A box plot was used to provide a convenient manner in which to visualize and compare the distributions of expression values for the two groups (a total of 8 samples) after normalization (Fig. 1A). Hierarchical clustering showed distinguishable circRNA expression profiling between the two groups (Fig. 1B). The Scatter-Plot provided a visualization method for reproducibility distinguishing the circRNA expression between the two compared groups, indicating that circRNA expression profiles in cholesteatoma differed markedly from those of normal skin tissues (Fig. 1C). A volcano plot was utilized to display the differentially expressed circRNAs with statistical significance between the cholesteatoma and normal skin groups (Fig. 1D). Therefore, by setting a threshold of fold change $>2.0$ and $\mathrm{P}<0.05$, a total of 355 significantly differentially expressed circRNAs were discriminated in cholesteatoma compared with normal skin tissues. Among these, 101 circRNAs were identified to be upregulated, whereas 254 were downregulated (fold change $>2.0, \mathrm{P}<0.05$ ). The microarray profile and RNAseq data sets have been deposited into Gene Expression Omnibus (GEO) with accession number GSE102715 (https://www.ncbi. nlm.nih.gov/geo/query/acc.cgi?acc=GSE102715).

Category characteristics of dysregulated circRNAs and their chromosomal distributions. In the present study, all the significantly differentially expressed circRNAs (fold change $>2.0, \mathrm{P}<0.05)$ were classified into five categories: Exonic (76\%), antisense (3\%), intronic (12\%), sense-overlapping (8\%), and intergenic (1\%). Among the upregulated circRNAs, 79 were exonic, 3 antisense, 12 intronic, and 7 sense-overlapping. Among the downregulated circRNAs, 189 were exonic, 9 antisense, 30 intronic, 22 sense-overlapping, and 4 intergenic. These results clearly demonstrated that exonic circRNAs account for the majority of all significantly differentially expressed circRNAs.

Furthermore, we also analyzed the chromosome distributions of all significantly differentially expressed circRNAs (fold change $>2.0, \mathrm{P}<0.05$ ) (Fig. 2). The dysregulated circRNAs originated from almost all human genomes, including chromosomes and the mitochondrial genome (chrM). For the upregulated circRNAs, 12 were located on chromosome 17 

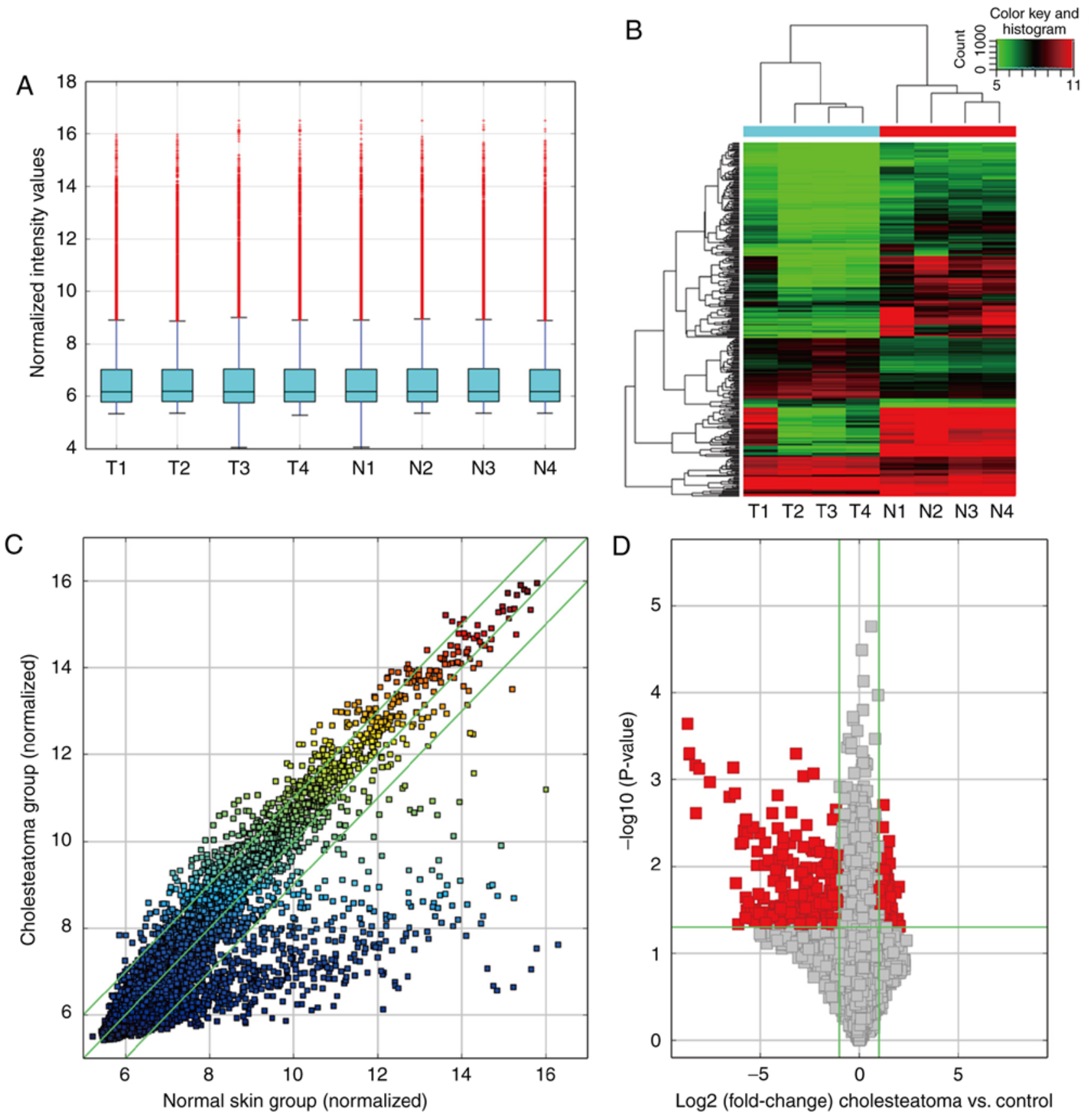

Figure 1. Microarray analysis of differentially expressed circRNAs in cholesteatoma samples vs. normal skin samples. (A) Box plot for convenient visualization and comparison of the distributions of expression values for the two groups after normalization. T1-T4: cholesteatoma groups; N1-N4: normal skin groups. (B) Hierarchical clustering to identify distinguishable circRNA expression profiling between cholesteatomas (T) and paired normal skin tissues (N). 'Red' and 'green' stand for high and low relative expression, respectively. (C) Scatter-Plot for visualization of the circRNA expression reproducibility (or variation) between cholesteatoma groups (treatment) and matched normal skin groups (control). $\mathrm{x}$ - and y-axes stand for the averaged normalized signal values of the group ( $\log 2$ scaled). Green lines stand for fold-change lines. (D) Volcano plot display of significantly differentially expressed circRNAs between the cholesteatoma (treatment) and matched normal skin (control) groups. Both magnitude of change and variability were taken into consideration. The green vertical lines correspond to 2.0-fold upregulation and downregulation, and the green horizontal line represents a P-value of 0.05 . circRNAs, circulating RNAs.

(chr17) and 9 on chr3 (Fig. 2A). Among the downregulated circRNAs, 22 were located on chrl and 21 on chr11 (Fig. 2B).

Microarray expression results are validated as highly reliable by quantitative reverse transcription-polymerase chain reaction $(R T-q P C R)$. To validate our microarray data, we randomly selected 6 circRNAs (fold change $>2$ and $\mathrm{P}<0.05$ from among 355 significantly dysregulated circRNAs for further validation in 7 pairs of cholesteatoma and matched skin tissues (4 pairs of original tissues and 3 another pairs of cholesteatoma and normal skin tissues) using RT-qPCR. As depicted in Fig. 3, the relative expression levels of validated circRNAs were consistent with those in the microarray data, which indicated our microarray analysis results as being highly reliable.

Gene ontology and pathway analyses suggest that circRNAs may regulate multiple biological functions in cholesteatoma 

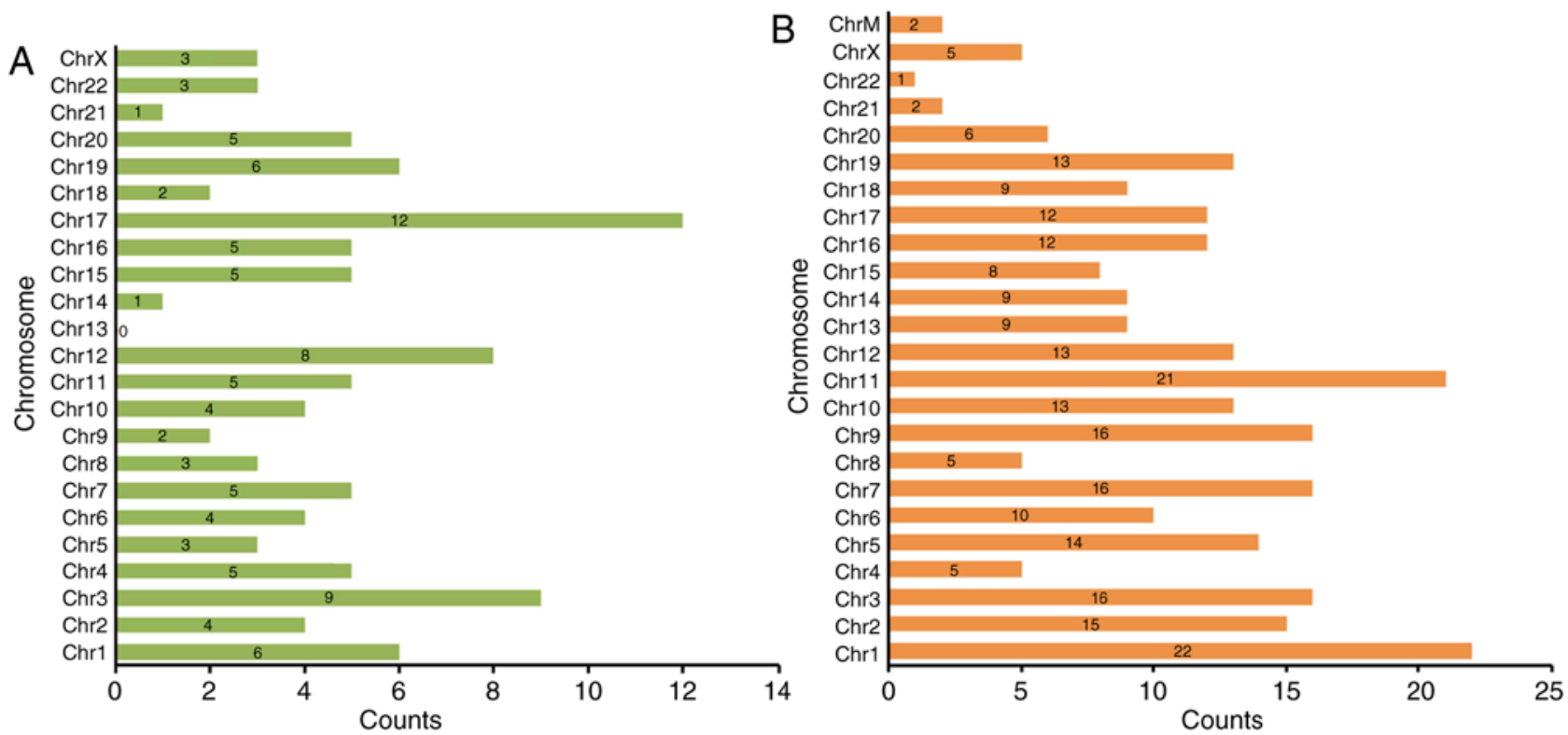

Figure 2. Chromosome distributions of all significantly differentially expressed circRNAs (fold change $>2.0, \mathrm{P}<0.05$ ). (A) Chromosome distributions of upregulated circRNAs. (B) Chromosome distributions of downregulated circRNAs. 'Count' stands for the number of significantly differentially expressed circRNAs. 'Chromosome' stands for different chromosomes. 'ChrM' stands for mitochondrial genome. circRNAs, circulating RNAs.

A
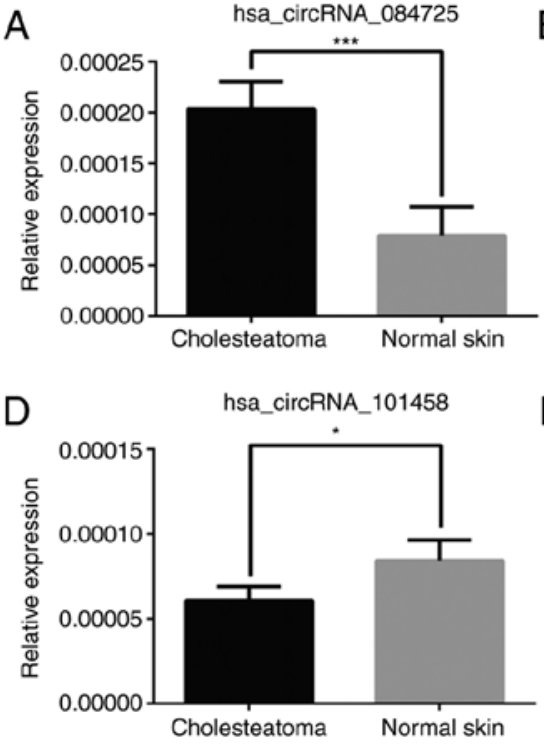

B

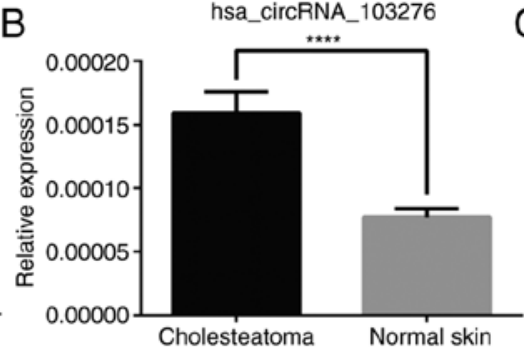

E

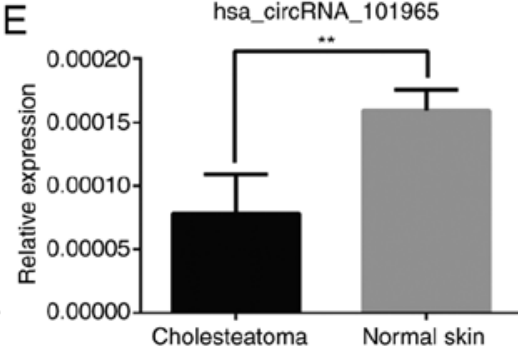

C

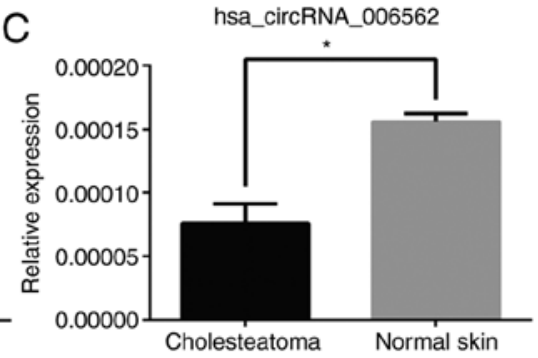

$\mathrm{F}$

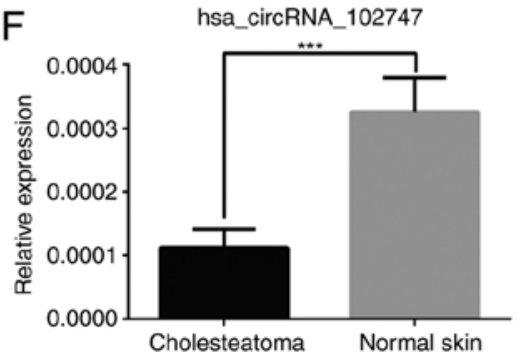

Figure 3. Validation of the microarray data by quantitative RT-PCR. Relative expression levels of dysregulated circRNAs are displayed comparing normal skins and cholesteatomas. (A) hsa_circRNA_084725; (B) hsa_circRNA_103276; (C) hsa_circRNA_006562; (D) hsa_circRNA_101458; (E) hsa_circRNA_101965; (F) hsa_circRNA_102747. Among them, A and B are upregulated circRNAs and C-F are downregulated circRNAs in cholesteatoma. Means \pm SD, "P<0.05, ${ }^{* *} \mathrm{P}<0.01,{ }^{* * *} \mathrm{P}<0.001$ and ${ }^{* * * *} \mathrm{P}<0.0001$, unpaired $\mathrm{t}$ test. circRNAs, circulating RNAs.

pathogenesis. CircRNAs are generated from the splicing of protein-coding genes and can regulate the functions of their parent genes. Therefore, to preliminarily understand the functions of circRNAs in cholesteatoma, we conducted gene ontology (GO) and pathway analyses of parent genes of the dysregulated circRNAs. We examined three aspects, biological process (BP), cellular component (CC), and molecular function (MF) in the GO analysis. Generally, 212 BP, 64 $\mathrm{CC}$, and $30 \mathrm{MF}$ GO terms were found to be significantly enriched $(\mathrm{P}<0.05)$ and the top-10 enriched GO terms of $\mathrm{BP}$, $\mathrm{CC}, \mathrm{MF}$ are listed (Fig. 4A-C). The majority of biological functions in the GO analysis consisted of cell morphogenesis, cell cycle, cell communication, stimulus response, and metabolic processes. By mapping the pathway analysis of parent genes to the Kyoto Encyclopaedia of Genes and Genomes (KEGG), 5 significantly enriched pathways were found in cholesteatoma $(\mathrm{P}<0.05$, Fig. 4D). These 5 pathways, glycosphingolipid biosynthesis, Th17 cell differentiation, galactose metabolism, Th1 and Th2 cell differentiation, and pyruvate metabolism, all correlated with cell growth, cell proliferation, cell migration, cell survival, and inflammation (29-33). 

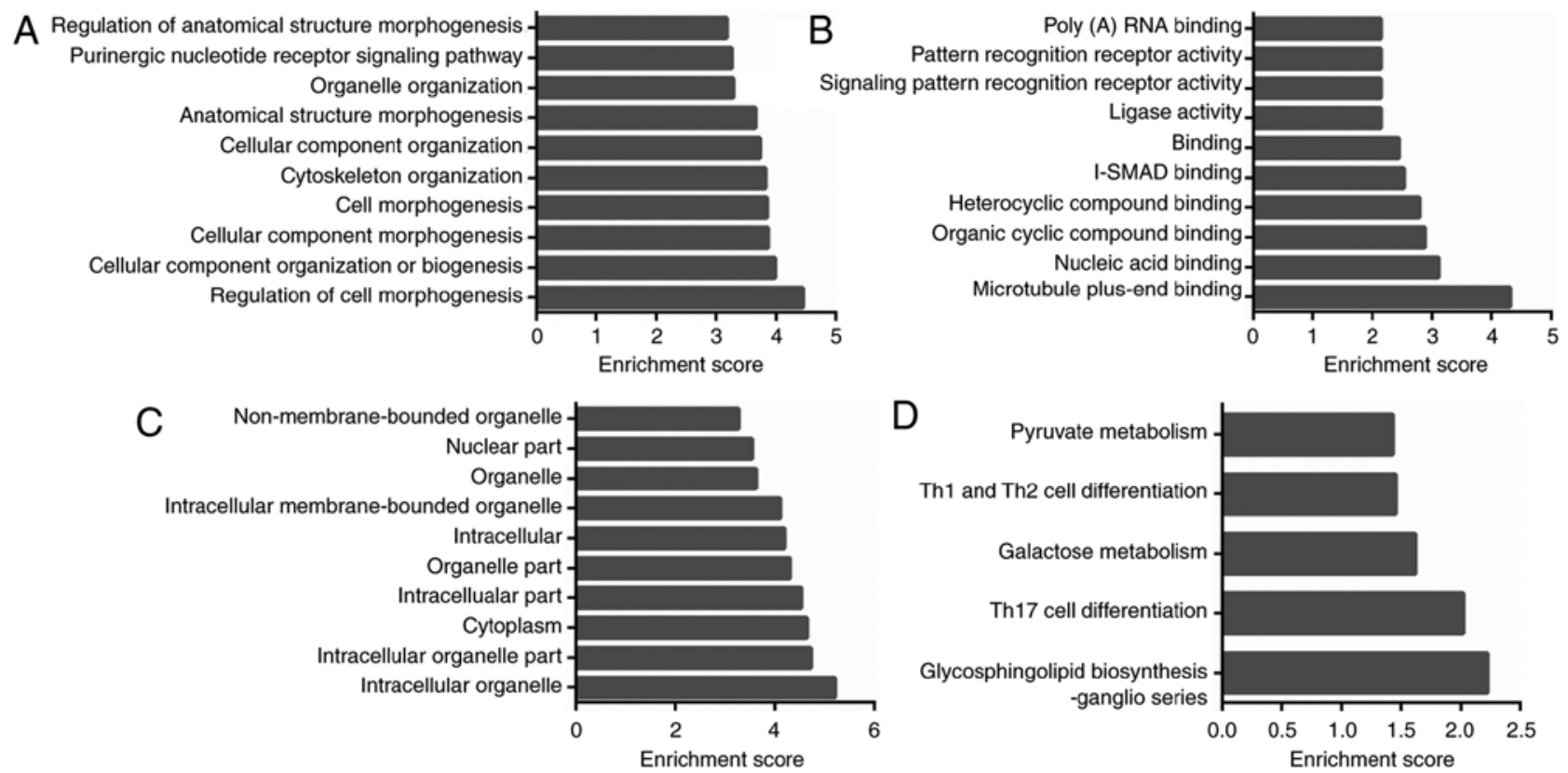

Figure 4. Functional analysis of circRNA parent genes. (A) The top-10 enriched biological process (BP) terms; (B) the top-10 enriched molecular function (MF) terms; (C) the top-10 enriched cellular component (CC) terms; (D) the 5 significantly enriched pathways. 'Enrichment score' stands for the enrichment score value of the GO ID, equivalent to - $\log 10$ (P-value). circRNAs, circulating RNAs.

ceRNA network analysis-elucidated circRNAs have ceRNA potential in cholesteatoma. According to the ceRNA hypothesis, all RNA transcripts communicate with each other by harboring multiple miRNA binding sites (i.e. MREs). In this model, MREs act as an 'RNA language' during the cross-talk of non-coding and coding RNAs (13). In our previous study, we confirmed that IncRNAs had ceRNA potential in cholesteatoma formation (15) (the microarray profile and RNAseq data sets have been deposited into GEO with accession number GSE102673 https://www.ncbi.nlm.nih.gov/geo/query/acc. cgi?acc $=$ GSE102673). Therefore, to explore the regulatory potential of circRNAs in the pathogenesis of cholesteatoma, we selected 2 significant differentially expressed circRNAs (hsa-circRNA-102747, hsa-circRNA-101458, fold change $>2.0, \mathrm{P}<0.05$, Table II), which shared common MREs with each other, to generate a circRNA-IncRNA-miRNA-mRNA ceRNA network (Fig. S1).

The network was shown to be composed of 2 circRNA nodes, 31 lncRNA nodes, 48 miRNA nodes and 248 mRNA nodes. GO and pathway analyses were also conducted for the ceRNA network. The results displayed there were a total of 520 significantly enriched GO terms $(\mathrm{P}<0.05)$ in the network, including $461 \mathrm{BP}, 17 \mathrm{CC}, 42 \mathrm{MF}$ terms and the top-10 enriched GO terms of BP, CC, MF are listed (Fig. 5A-C). In the GO analysis, we identified that almost all the GO processes were closely related with various metabolic processes. In the KEGG pathway analysis, 24 enriched pathways were discovered, of which the MAPK signaling pathway (hsa04010) has previously drawn the attention of researchers owing to its function in cancer (34) (Fig. 5D).

In the network, we noted that circRNA-102747 interacted with miR-21-3p (Figs. 5E and 6), a microRNA belonging to the miR-21 family, which has been confirmed to promote the formation and invasion of cholesteatoma $(7,8)$. Furthermore,
circRNA-101458 was found to interact with miR let-7a-3p (Figs. 5F and 7), a microRNA belonging to the miR let-7a family, the upregulation of which has been considered to have an antiproliferative effect and contribute to the benign nature of cholesteatoma $(11,35)$. The complementarity between the 2 candidate circRNAs and the 2 miRNAs was perfect according to 7 mer-m8 matching types (Fig. 5E and F). In addition, in the circRNA-102747-mediated ceRNA network, circRNA-102747 and IncRNA-uc001kfc. 1 both interacted with miR-21-3p (Fig. 6); of which IncRNA-uc001kfc.1 was considered to play a key role in cholesteatoma pathogenesis in our previous research study (15).

\section{Discussion}

In the present study, by microarray analysis, we discovered that circulating RNA (circRNA) expression profiles in cholesteatoma were significantly dysregulated compared with those in normal skin tissues with 101 circRNAs upregulated and 254 downregulated, indicating that circRNAs may play crucial roles in cholesteatoma. In the category analysis of differentially expressed circRNAs, exonic circRNAs accounted for the largest type by $76 \%$. This is likely functionally relevant, because exonic circRNAs are derived from exonic regions within coding genes directly through back splicing by covalently linking the $3^{\prime}$ end of an exon with the $5^{\prime}$ end of either the same exon or the upstream exon. This signature structure protects exonic circRNAs from 'exon skipping' and renders them more inclined to regulate the linear coding RNAs from which they are generated (36-39). In the chromosome distribution analysis, we found that differentially expressed circRNAs were more inclined to be located on chr17, chrl, and chr11. Furthermore, Ecsedi et al demonstrated that chromosomal imbalances play an important role in cell proliferation activation 
Table II. Significantly differentially expressed circRNAs for ceRNA network construction (fold change $>2.0, \mathrm{P}<0.05$ ).

\begin{tabular}{llccr}
\hline circRNA & Gene symbol & Fold change & P-value & Regulation \\
\hline hsa_circRNA_102747 & ACTR2 & 2.32100 & 0.0190592 & Downregulated \\
hsa_circRNA_101458 & HERC2P3 & 27.86245 & 0.0380877 & Downregulated
\end{tabular}

circRNAs, circulating RNAs; ceRNA, competing endogenous RNA; ACTR2, actin related protein 2; HERC2P3, hect domain and RLD 2 pseudogene 3 .

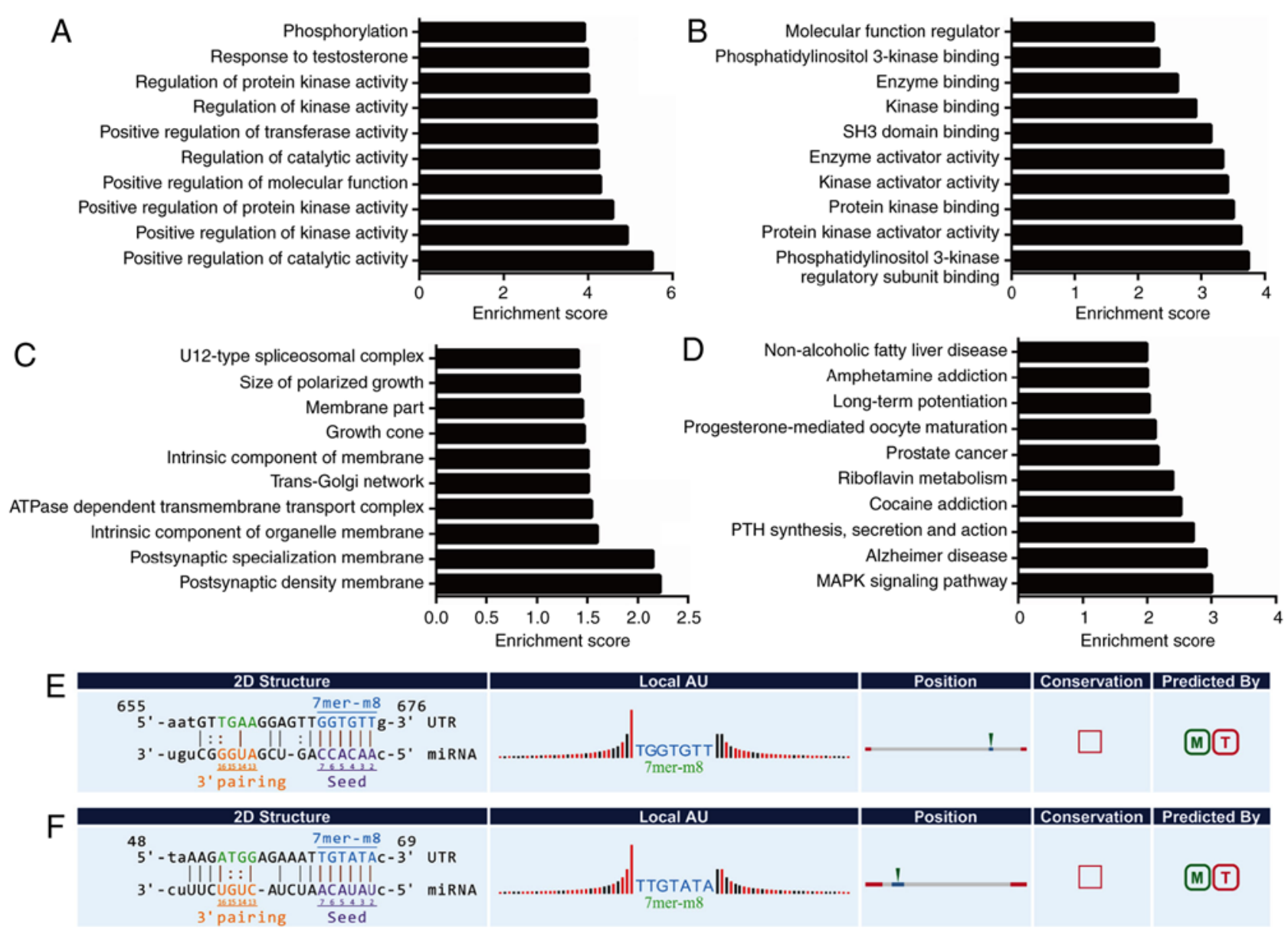

Figure 5. Enriched functional terms in the circRNA-lncRNA-miRNA-mRNA ceRNA network and 2D structure of miRNA vs. circRNA. (A-C) The top-10 significantly enriched Gene Ontology (GO) terms in the circRNA-lncRNA-miRNA-mRNA ceRNA network of cholesteatoma (P<0.05). (A) biological process; (B) molecular function; (C) cellular component. 'Enrichment score' stands for the enrichment score value of the GO ID, equivalent to -log10(P-value). (D) The top-10 significantly enriched Kyoto Encyclopedia of Genes and Genomes (KEGG) terms in the circRNA-lncRNA-miRNA-mRNA ceRNA network of cholesteatoma $(\mathrm{P}<0.05)$. 'Enrichment score' stands for the enrichment score value of the Pathway ID, equivalent to '-log10(P-value)'. (E) The 2D structure of miR-21-3p vs. circRNA-102747. (F) The 2D structure of miR let-7a-3p vs. circRNA-101458. The 2D structure shows the specific location of the binding sites on the full-length sequence of the circRNA. The type of binding site is 7mer-m8, indicating perfectly matched positions 2 to 8 of the mature miRNA; 'l' indicates an exact match; ' $:$ ' indicates G: U pairing. Local AU indicates accessibility of binding sites; the red bar indicates that the location is A:U; the darker red bar indicates higher weighing of A:U. Position: relative position of binding sites on circRNA. Conservation: relative conservation of the seed complementary region between species. However, there are insufficient data for constructing a phylogenetic tree of different species for circRNAs. Predicted by: 'M' stands for miRanda; ' $T$ ' stands for TargetScan. circRNA, circulating RNA; IncRNA, long non-coding RNA; miRNA, microRNA; mRNA, messenger RNA; ceRNA, competing endogenous RNA.

and bone invasion (40); thus, our finding may partially support this report. In addition, the RT-qPCR results were verified to coincide with the microarray data, which indicated that the microarray analysis was highly reliable. Together, these circRNA profile analyses suggest that circRNAs have regulatory function potential in the epigenetic regulatory mechanism of cholesteatoma formation.
To preliminarily understand the functions of circRNAs in cholesteatoma, we performed functional analysis on the parental genes of the dysregulated circRNAs in cholesteatoma. This revealed that the majority of related biological processes involved cell morphogenesis, cell cycle, cell communication, stimulus response, and metabolic processes. Pathway analysis elucidated that 'glycosphingolipid biosynthesis', 'Th17 cell 


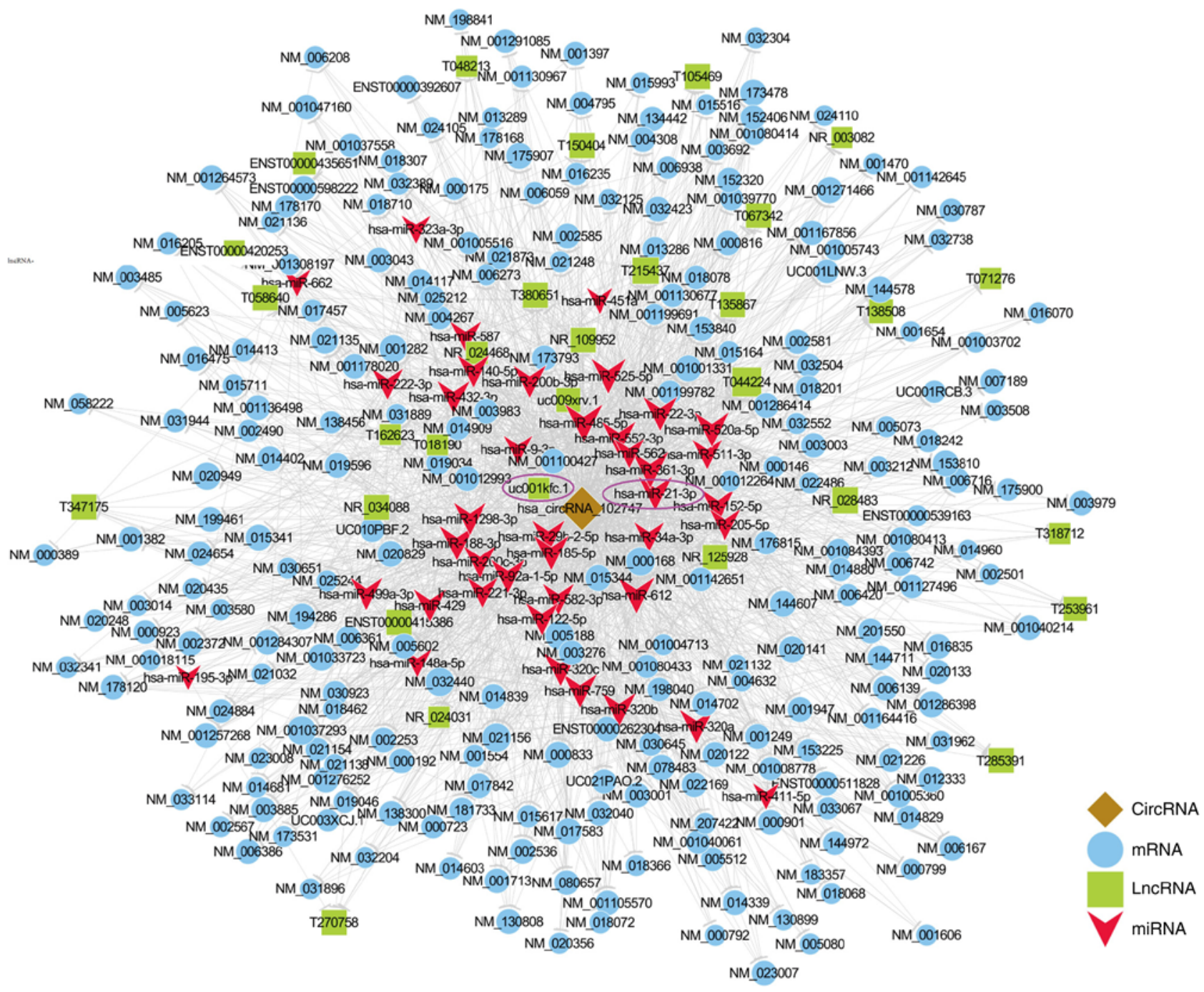

Figure 6. circRNA-102747-mediated circRNA-IncRNA-miRNA-mRNA ceRNA network. The network was based on circRNA-lncRNA-miRNA-mRNA interactions. In the network, hsa-miR-21-3p, which shares microRNA response element with both circRNA-102747 and lncRNA-uc001kfc.1, was verified to play a vital role in cholesteatoma. Both hsa-miR-21-3p and lncRNA-uc001kfc.1 are circled in purple. Blue, mRNA; green, IncRNA; red, miRNA. circRNA, circulating RNA; lncRNA, long non-coding RNA; miRNA, microRNA; mRNA, messenger RNA; ceRNA, competing endogenous RNA.

differentiation', 'galactose metabolism', 'Th1 and Th2 cell differentiation', and 'pyruvate metabolism' were all enriched, which correlate with cell growth, cell proliferation, cell migration, cell survival, and inflammation associations (29-33). Considering that cholesteatoma is a disease caused by the hyper-proliferation of keratinocytes, in the present study, the functional analyses of the parental genes of dysregulated circRNAs together implied that circRNAs may contribute to cholesteatoma formation.

According to the competing endogenous RNA (ceRNA) hypothesis, multiple miRNA binding sites (MREs) can act as an 'RNA language' during the cross-talk of non-coding and coding RNAs (13). To verify our hypothesis and explore whether circRNAs could function as ceRNAs in the pathogenesis of cholesteatoma, we selected 2 significantly differentially expressed circRNAs (circRNA-102747, circRNA-101458, fold change $>2.0, \mathrm{P}<0.05$ ), which shared common MREs with each other, to generate a circRNA-IncRNA-miRNA-mRNA ceRNA network (Fig. S1). Functional analysis of the ceRNA network revealed that multiple enriched GO processes in the network were associated with various metabolic processes, such as protein kinase activity, phosphatidylinositol 3-kinase (PI3K) binding. PI3Ks are enzymes that catalyze the phosphorylation of phosphatidylinositol (PtdIns). The PI3K pathway plays a key role in the regulation of cell survival and proliferation (41). A previous study reported that activation of the PI3K/Akt (Akt, i.e. serine kinase PKB, a downstream effector of PI3K) signaling pathway protects epithelial keratinocytes of cholesteatoma against programmed cell death (42). Moreover, increased PI3K/Akt signaling pathway activation has been proven to be related to cholesteatoma epithelial hyper-proliferation $(43,44)$. In the KEGG pathway analysis of the ceRNA network, the most enriched pathway was found to be the MAPK (mitogen-activated protein kinase) signaling pathway, activation of which has previously been proven to play an important role in the terminal differentiation in cholesteatoma epithelium (42).

In the network, we noted that circRNA-102747 and lncRNA-uc001kfc. 1 both interacted with miR-21-3p, and lncRNA-uc001kfc.1 was downregulated in cholesteatoma 


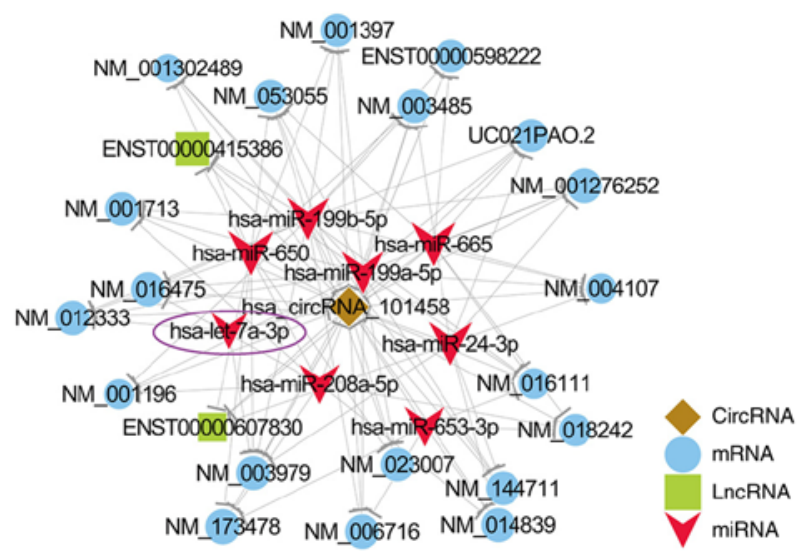

Figure 7. circRNA-101458-mediated circRNA-lncRNA-miRNA-mRNA ceRNA network. In the network, miR let-7a-3p, which shares microRNA response element with both circRNA-101458, was verified to play a vita role in cholesteatoma. miR let-7a-3p is circled in purple. Blue, mRNA green, lncRNA; red, miRNA. circRNA, circulating RNA; lncRNA, long non-coding RNA; miRNA, microRNA; mRNA, messenger RNA; ceRNA, competing endogenous RNA.

and was considered to function as an 'endogenous sponge' for miR-21-3p in cholesteatoma pathogenesis in our previous research (15). In the present study, compared with normal skin tissues, circRNA-102747 was also confirmed to have low expression in cholesteatoma by both microarray analysis and RT-qPCR. The 2D structure of circRNA-102747 and miR-21-3p showed that the complementarity between them was perfect with the miRNA seed sequence AACACC. Furthermore, our previous study indicated that the binding site of miR-21-3p on 1 ncRNA-uc001kfc. 1 was also perfectly matching with the same miRNA seed sequence AACACC (15). The miRNA seed sequence, which is the nucleotides 2-7 of the 5' region of the miRNA and considered as the most conserved portion of the miRNAs, is particularly important for miRNA recognition $(12,45)$. The perfectly matching types with the same miRNA seed sequence further confirmed that both circRNA-102747 and lncRNA-uc001kfc. 1 could share the same MRE with miR-21-3p. Therefore, we presume that by using the MRE as 'RNA language' (13), circRNA-102747, together with 1ncRNA-uc001kfc.1, functions as an 'endogenous sponge' for miR-21-3p. In previous studies, miR-21 has been proven to be upregulated in cholesteatoma (7). Overexpression of miR-21 was found to promote cell proliferation and invasion of keratinocytes, which contributed to the malignant nature of cholesteatoma $(7,11)$. In the crosstalk of circRNA-102747/1ncRNA-uc001kfc.1/miR-21-3p/targeted mRNAs, when circRNA-102747 and lncRNA-uc001kfc. 1 are downregulated in cholesteatoma, hsa-miR-21-3p becomes transcriptionally active and thus regulates targeted genes and results in the hyper-proliferation of keratinocytes.

In the circRNA-101458-mediated ceRNA network, we found that circRNA-101458 interacted with miR let-7a-3p, a microRNA belonging to the $\mathrm{miR}$ let-7a family. The upregulation of miR let-7 can suppress cancer development by targeting specific oncogenes and is considered to have an antiproliferative effect $(35,46)$. As is known, cholesteatoma is manifested by certain malignant characteristics, such as excessive squamous epithelial cell proliferation. However, cholesteatoma pathologically displays benign tumors without abnormal keratinocyte mitosis. In previous research, miR let-7a has been confirmed to be upregulated in cholesteatomas and contribute to the benign nature of cholesteatoma (11). In the present study, circRNA-101458 was found to be significantly downregulated in cholesteatoma. Herein we presume that circRNA-101458 may play a key role in cholesteatoma pathogenesis and function as an 'endogenous sponge' for let-7a-3p; in the crosstalk of circRNA-101458/miR let-7a-3p/targeted mRNAs, when circRNA-101458 is downregulated in cholesteatoma, miR let-7a-3p becomes transcriptionally active and thus regulating targeted genes resulting in the anti-proliferation of keratinocytes in cholesteatoma. Therefore, we formed the hypothesis that in cholesteatoma, the circRNA-102747/ lncRNA-uc001kfc. $1 / \mathrm{miR}-21-3 \mathrm{p} /$ targeted mRNAs may act as the regulator of malignant characteristics, whereas circRNA-101458/miR let-7a-3p/targeted mRNAs function as a benign regulator. This suggests that circRNA-102747 and circRNA-101458 have ceRNA potential in the pathogenesis of cholesteatoma and can be potentially therapeutic targets for the drug therapy of cholesteatoma. In future studies, we will focus on the functional studies and elucidate the ceRNA-mediated effects of circRNAs in the pathogenesis of cholesteatoma.

Although we put forward the assumption that the circRNA-miRNA-targeted mRNAs axis may be involved in the mechanism of cholesteatoma pathogenesis, some limitations must be considered in the present study. Firstly, the sample size in the study was relatively small and the scale of circRNA profile might be decreased with larger number of subjects. However, as there are few similar studies and it is the first study of circRNAs in cholesteatoma, it adds important knowledge to the current field. Secondly, we only studied the circRNA profiles and their ceRNA potential in the pathogenesis of cholesteatoma, while the functional analysis is imperfect. Functional research, such as selectively upregulating or downregulating the expression of certain circRNAs in vitro or in vivo, are needed to validate the exact role of circRNAs in cholesteatoma pathogenesis in future studies.

In conclusion, our study elucidates the profiles of circRNAs in cholesteatoma for the first time by using microarray analysis. Significantly differentially expressed circRNAs were found in cholesteatoma compared with the normal skin group and their functions were predicted through GO and pathway analyses, the results of which together indicate that circRNAs may contribute to the pathogenesis of cholesteatoma. By constructing circRNA-mediated ceRNA networks, we found that circRNAs may function as ceRNAs that could sequester targeted miRNAs and influence the associated gene functions. circRNAs may thus constitute promising therapeutic agents for cholesteatoma. In future studies, we will focus on the functional analysis of circRNAs to explore the precise molecular mechanisms of cholesteatoma.

\section{Acknowledgements}

Not applicable.

\section{Funding}

No funding was received. 


\section{Availability of data and materials}

All data generated or analyzed during the present study are included in this published article or are available from the corresponding author on reasonable request. The accession number for circRNA-seq data in this study is GSE102715 (https://www. ncbi.nlm.nih.gov/geo/query/acc.cgi?acc=GSE102715).

\section{Authors' contributions}

Conception and design of the study was carried out by HY and JG. JG, QT, RX, XZ, SW, YZ and WL performed the experiments. Acquisition of the data was carried out by JG and HY. Analysis and interpretation of the data were the responsibility of JG and HY. Reagents/materials/analysis tools were procured by JG, SW, ZG and HY. Drafting of the article and revising it critically for important intellectual content was carried out by JG and HY. Final approval of the version was submitted by HY. All authors read and approved the manuscript and agree to be accountable for all aspects of the research in ensuring that the accuracy or integrity of any part of the work are appropriately investigated and resolved.

\section{Ethics approval and consent to participate}

All tissue donors participating in this study provided written informed consent. Ethical approval for our study was obtained from the Ethics Committee of Peking Union Medical College Hospital (no. S-K292).

\section{Patient consent for publication}

Not applicable.

\section{Competing interests}

The authors declare that they have no competing financial interests.

\section{References}

1. Louw L: Acquired cholesteatoma pathogenesis: Stepwise explanations. J Laryngol Otol 124: 587-593, 2010.

2. Britze A, Moller ML and Ovesen T: Incidence, 10-year recidivism rate and prognostic factors for cholesteatoma. J Laryngol Otol 131: 319-328, 2017.

3. Ergun S, Zheng X and Carlsoo B: Expression of transforming growth factor-alpha and epidermal growth factor receptor in middle ear cholesteatoma. Am J Otol 17: 393-396, 1996.

4. Olszewska E, Wagner M, Bernal-Sprekelsen M, Ebmeyer J, Dazert S, Hildmann H and Sudhoff H: Etiopathogenesis of cholesteatoma. Eur Arch Otorhinolaryngol 261: 6-24, 2004.

5. Kojima H, Shiwa M, Kamide Y and Moriyama H: Expression and localization of mRNA for epidermal growth factor and epidermal growth factor receptor in human cholesteatoma. Acta Otolaryngol 114: 423-429, 1994.

6. Kupper TS: The activated keratinocyte: A model for inducible cytokine production by non-bone marrow-derived cells in cutaneous inflammatory and immune responses. J Invest Dermatol 94 (6 Suppl): 146s-150s, 1990.

7. Friedland DR, Eernisse R, Erbe C, Gupta N and Cioffi JA: Cholesteatoma growth and proliferation: Posttranscriptional regulation by microRNA-21. Otol Neurotol 30: 998-1005, 2009.

8. Chen X, Li X and Qin Z: MicroRNA-21 promotes the proliferation and invasion of cholesteatoma keratinocytes. Acta Otolaryngol 136: 1261-1266, 2016
9. Zhang W, Chen X and Qin Z: MicroRNA let-7a suppresses the growth and invasion of cholesteatoma keratinocytes. Mol Med Rep 11: 2097-2103, 2015.

10. Li N and Qin ZB: Inflammation-induced miR-802 promotes cell proliferation in cholesteatoma. Biotechnol Lett 36: 1753-1759, 2014.

11. Chen X and Qin Z: Post-transcriptional regulation by microrna-21 and let-7a microRNA in paediatric cholesteatoma. J Int Med Res 39: 2110-2118, 2011.

12. Bartel DP: MicroRNAs: Target recognition and regulatory functions. Cell 136: 215-233, 2009.

13. Salmena L, Poliseno L, Tay Y, Kats L and Pandolfi PP: A ceRNA hypothesis: The Rosetta Stone of a hidden RNA language? Cell 146: 353-358, 2011

14. Bak RO and Mikkelsen JG: miRNA sponges: Soaking up miRNAs for regulation of gene expression. Wiley Interdiscip Rev RNA 5: 317-333, 2014.

15. Gao J, Tang Q, Zhu X, Wang S, Zhang Y, Liu W, Gao Z and Yang H: Long noncoding RNAs show differential expression profiles and display ceRNA potential in cholesteatoma pathogenesis. Oncol Rep 39: 2091-2100, 2018.

16. Jeck WR, Sorrentino JA, Wang K, Slevin MK, Burd CE, Liu J, Marzluff WF and Sharpless NE: Circular RNAs are abundant, conserved, and associated with ALU repeats. RNA 19: 141-157, 2013.

17. Memczak S, Jens M, Elefsinioti A, Torti F, Krueger J, Rybak A, Maier L, Mackowiak SD, Gregersen LH, Munschauer M, Loewer A, et al: Circular RNAs are a large class of animal RNAs with regulatory potency. Nature 495: 333-338, 2013.

18. Salzman J, Gawad C, Wang PL, Lacayo N and Brown PO: Circular RNAs are the predominant transcript isoform from hundreds of human genes in diverse cell types. PLoS One 7: e30733, 2012.

19. Starke S, Jost I, Rossbach O, Schneider T, Schreiner S, Hung LH and Bindereif A: Exon circularization requires canonical splice signals. Cell Rep 10: 103-111, 2015.

20. Guo JU, Agarwal V, Guo H and Bartel DP: Expanded identification and characterization of mammalian circular RNAs. Genome Biol 15: 409, 2014.

21. Hansen TB, Jensen TI, Clausen BH, Bramsen JB, Finsen B, Damgaard CK and Kjems J: Natural RNA circles function as efficient microRNA sponges. Nature 495: 384-388, 2013.

22. Huang M,Zhong Z,Lv M,Shu J,Tian Q and Chen J: Comprehensive analysis of differentially expressed profiles of lncRNAs and circRNAs with associated co-expression and ceRNA networks in bladder carcinoma. Oncotarget 7: 47186-47200, 2016.

23. Fan X, Weng X, Zhao Y, Chen W, Gan T and Xu D: Circular RNAs in Cardiovascular Disease: An Overview. Biomed Res Int 2017: 5135781, 2017.

24. Zhang S,Zhu D, Li H,LiH,Feng C and Zhang W: Characterization of circRNA-Associated-ceRNA networks in a senescence-accelerated mouse prone 8 brain. Mol Ther 25: 2053-2061, 2017.

25. Ritchie ME, Phipson B, Wu D, Hu Y, Law CW, Shi W and Smyth GK: limma powers differential expression analyses for RNA-sequencing and microarray studies. Nucleic Acids Res 43: e47, 2015.

26. Livak KJ and Schmittgen TD: Analysis of relative gene expression data using real-time quantitative PCR and the 2(-Delta Delta C(T)) method. Methods 25: 402-408, 2001.

27. Pasquinelli AE: MicroRNAs and their targets: Recognition, regulation and an emerging reciprocal relationship. Nat Rev Genet 13: 271-282, 2012.

28. Enright AJ, John B, Gaul U, Tuschl T, Sander C and Marks DS: MicroRNA targets in Drosophila. Genome Biol 5: R1, 2003.

29. D'Angelo G,Capasso S, Sticco L and Russo D: Glycosphingolipids: Synthesis and functions. FEBS J 280: 6338-6353, 2013.

30. Patel DD and Kuchroo VK: Th17 Cell Pathway in Human Immunity: Lessons from genetics and therapeutic interventions. Immunity 43: 1040-1051, 2015.

31. Gray LR, Tompkins SC and Taylor EB: Regulation of pyruvate metabolism and human disease. Cell Mol Life Sci 71: 2577-2604, 2014.

32. Coelho AI, Berry GT and Rubio-Gozalbo ME: Galactose metabolism and health. Curr Opin Clin Nutr Metab Care 18: 422-427, 2015.

33. Kidd P: Th1/Th2 balance: The hypothesis, its limitations, and implications for health and disease. Altern Med Rev 8: 223-246, 2003.

34. Kim EK and Choi EJ: Pathological roles of MAPK signaling pathways in human diseases. Biochim Biophys Acta 1802: 396-405, 2010. 
35. Johnson CD, Esquela-Kerscher A, Stefani G, Byrom M, Kelnar K Ovcharenko D, Wilson M, Wang X, Shelton J, Shingara J, et al: The let-7 microRNA represses cell proliferation pathways in human cells. Cancer Res 67: 7713-7722, 2007.

36. Jeck WR and Sharpless NE: Detecting and characterizing circular RNAs. Nat Biotechnol 32: 453-461, 2014.

37. Ashwal-Fluss R, Meyer M, Pamudurti NR, Ivanov A, Bartok O, Hanan M, Evantal N, Memczak S, Rajewsky N and Kadener S: circRNA biogenesis competes with pre-mRNA splicing. Mol Cell 56: 55-66, 2014.

38. Lasda E and Parker R: Circular RNAs: Diversity of form and function. RNA 20: 1829-1842, 2014.

39. Ebbesen KK, Hansen TB and Kjems J: Insights into circular RNA biology. RNA Bio 14: 1035-1045, 2017.

40. Ecsedi S, Rakosy Z, Vizkeleti L, Juhász A, Sziklai I, Adány R and Balázs M: Chromosomal imbalances are associated with increased proliferation and might contribute to bone destruction in cholesteatoma. Otolaryngol Head Neck Surg 139: 635-640, 2008.

41. Song G, Ouyang G and Bao S: The activation of Akt/PKB signaling pathway and cell survival. J Cell Mol Med 9: 59-71, 2005.
42. Huisman MA, De Heer E and Grote JJ: Survival signaling and terminal differentiation in cholesteatoma epithelium. Acta Otolaryngol 127: 424-429, 2007.

43. Yune TY and Byun JY: Expression of PTEN and phosphorylated Akt in human cholesteatoma epithelium. Acta Otolaryngol 129: 501-506, 2009.

44. Hawkins PT, Anderson KE, Davidson K and Stephens LR: Signalling through Class I PI3Ks in mammalian cells. Biochem Soc Trans 34: 647-662, 2006.

45. Lim LP, Lau NC, Weinstein EG, Abdelhakim A, Yekta S, Rhoades MW, Burge CB and Bartel DP: The microRNAs of Caenorhabditis elegans. Genes Dev 17: 991-1008, 2003.

46. Park SM, Shell S, Radjabi AR, Schickel R, Feig C, Boyerinas B, Dinulescu DM, Lengyel E and Peter ME: Let-7 prevents early cancer progression by suppressing expression of the embryonic gene HMGA2. Cell Cycle 6: 2585-2590, 2007.

(C) (1) () This work is licensed under a Creative Commons EY NO ND Attribution-NonCommercial-NoDerivatives 4.0 International (CC BY-NC-ND 4.0) License. 